\title{
A workbench for species identification based on images and deep learning techniques
}

\author{
Ignacio Heredia ${ }^{\ddagger}$, Lara Lloret ${ }^{\ddagger}$, Jesús Marco ${ }^{\ddagger}$, Francisco Pando§ \\ ‡ Instituto de Fisica de Cantabria -UNICAN CSIC, Santander, Spain \\ $\S$ Real Jardin Botanico -CSIC, Madrid, Spain
}

Corresponding author: Francisco Pando (pando@rjb.csic.es)

Received: 24 Aug 2017 | Published: 24 Aug 2017

Citation: Heredia I, Lloret L, Marco J, Pando F (2017) A workbench for species identification based on images and deep learning techniques. Proceedings of TDWG 1: e20569.

https://doi.org/10.3897/tdwgproceedings.1.20569

\section{Abstract}

We are currently studying the feasibility of applying deep learning techniques to natural sciences. In this contribution we will show our recent advances with an easy plug-and-play framework (that uses the Lasagne module built on top of Theano), which we have successfully trained for plant identification. Subsequent trials have been carried out on cone snails (Conus spp.) with minimum overhead and without writing any new code.

The fact that these applications share a common API makes it very easy to create new applications (e.g., on Android, as we are currently testing) and to apply them to new species groups. The code for the framework can be found at: https://github.com/ IgnacioHeredia/plant classification.

This kind of application makes taxonomic expertise directly accessible to members of the general public interested in nature and the diversity of living organisms. These applications have a clear educational impact, and may also be used to enhance conservation actions.

Deployment and use of the currrent framework is supported by the recently begun, EUfunded project "DEEP Hybrid Datacloud". In particular, the project will support the extensive training of the system needed to develop new applications, and will provide the necessary computational resources to the users. 


\section{Keywords}

Deep learning, neural networks, image recognition

\section{Presenting author}

Francisco Pando 